\title{
The Research of a Laser Micro Displacement Measuring System Based on the Spectral Interference of Optic Fiber Principle
}

\author{
Hui Zhang ${ }^{1,}$, Jun Shen ${ }^{1, b}$ and Wei Wang ${ }^{1, c}$ \\ ${ }^{1}$ Hefei University of Technology, Hefei 230009, China;

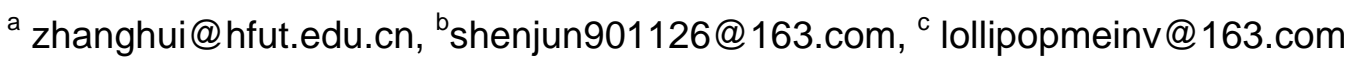

Keywords: the two-beam interference of a single fiber, athermalization probe, phase difference and displacement, accuracy, range

Abstract. In this article, a design of a laser micro displacement measuring system based on the spectral interference of optic fiber principle is presented. This system makes the transmission and interference of laser occur in a single fiber. At the same time, a kind of athermalization probe based on the wedge-shaped fiber and angulated to the fiber is designed, improving the anti-interference ability of the system. Besides, a model of the spectral interference of optical fiber system with the center wavelength of $1550 \mathrm{~nm}$ has been set up. The measurement accuracy of the system can research $300 \mathrm{~nm}$ and the measurement range can research around $1 \mathrm{~mm}$. The system not only has good anti-interference ability but also solves the problem of the coexistence of the nanometer-level measurement accuracy and the large-span measurement range.

\section{Introduction}

With the development of micro and nano technology at home and abroad and the rapid rise of optical fiber sensing technology of our country, the optical interference measurement has attracted much attention because of its advantages such as high resolution and non-contact characteristic. But the measurement also has two problems. One is that it is easily interfered by the environment and the other is that the measurement range is limited by the wavelength. Based on the Michelson interference measuring technology of the dual-wavelength fiber, the measuring system of the spectral interference of optic fiber discussed in the article makes the measurement beam and the reference beam travel and interfere through the same polarization maintain fiber(PMF), which not only improves the anti-interference ability of the system but also ensures good measurement accuracy and range of the system in the way of two-wavelength interference through a single fiber.

\section{The Principle of Spectral Interference}

The Principle of Spectral Interference: a laser beam emitted from a laser light source is divided through the fiber optic coupler into two beams one of which shines on the sample and then reflects and the other shines on the reference mirror and then reflects. The two reflected beams interfere in the fiber optic coupler and this interference is Michelson interference. When the sample moves, its displacement variation is calculated through the change of light intensity of the interfered beam[1, 2].

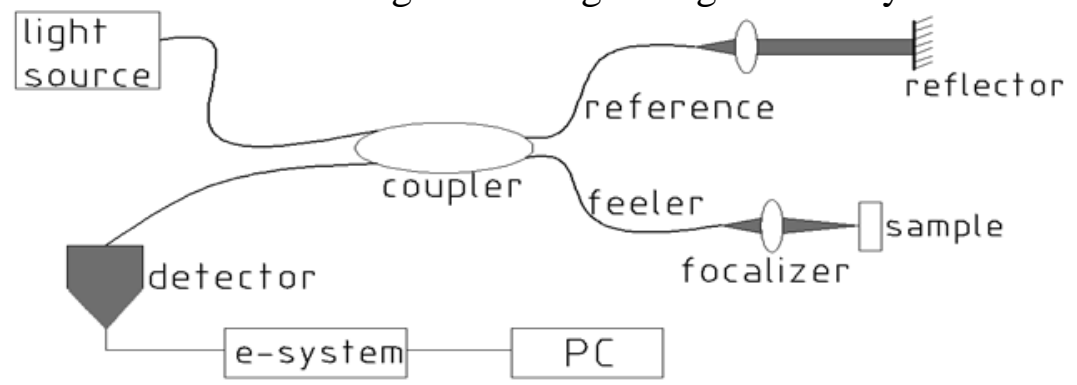

Fig. 1 Dual-fiber interference 


\section{The Introduction of the System}

The Principle of the System. The spectral interference of optic fiber system in this article, based on the dual-wavelength optic fiber interference, makes the transmission and interference of laser occur in a single fiber and there are no electronics within the measuring probe so there is no heating phenomenon in it, making the system apply in more occasions, besides, improving the anti-interference ability of the system[3].

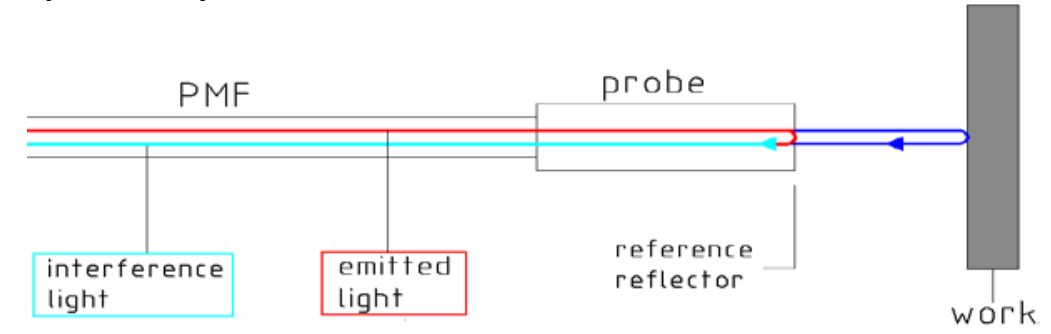

Fig. 2 Single fiber interference

In the system, the infracted light emitted by SLD (Super-luminescent diode) goes into the optical fiber polarizer after being aligned and focused by L1 and L2 and then the light goes into the PMF in the form of linear polarized light[4]. Then the light goes into the coupler through port1 and then goes out through port 2 and then it strikes on the sample to be tested by the probe. The end face of the probe has been processed so it has an inclination of $3^{\circ}$ and at the same time, its both sides have been processed into wedge shape. The processed probe can reflect and focus light[5]. The two beams reflected by the reference surface and the sample to be tested interfere in a single optical fiber and then go out through port3 of the coupler. After aligned by optical fiber collimator, the light output by port3 of the coupler strikes on the reflective diffraction grating and then the diffracted light strikes on the concave mirror which focuses the spectral light on the spectroscope. Through the spectroscope and linear array CCD, the change of light intensity with different wavelengths is transformed into the change of voltage value and then output. According to the output voltage value processed by the computer data acquisition system, the displacement change of the object to be tested can be got[6]. The structure of the measuring system is shown in figure 3 and the optical fiber is shown in bold.

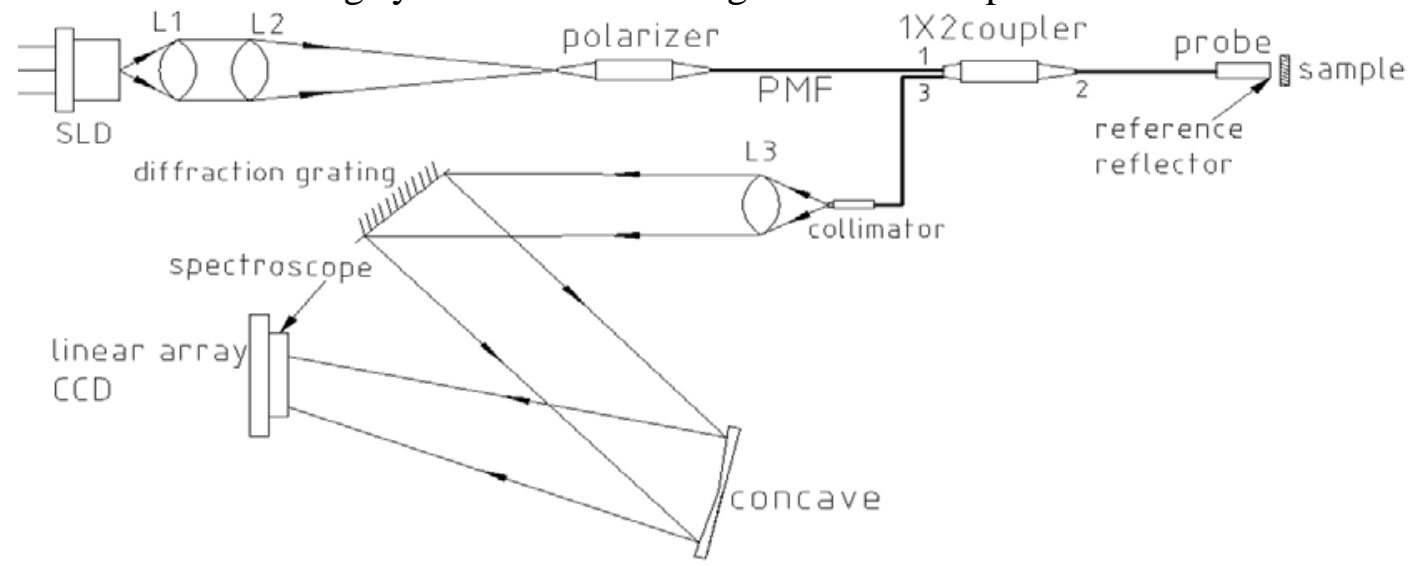

Fig. 3 The structure of the measuring system

The Structure of the Athermalization Probe. As shown in figure4, the probe designed in this article is based on the SMF (single mode fiber). First, the rear of the SMF is processed into wedge shape and then the wedge-shaped face is grinded into a face with an inclination of $3^{\circ}$. Figure 1 is SMF, figure2 is wedge-shaped optical fiber and figure3 is the end face of optical fiber.

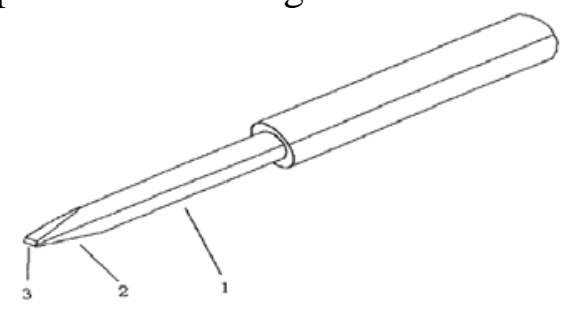

Fig. 4 The Structure of the Athermalization Probe 


\section{The Calculation of Accuracy and Range}

The Measuring Accuracy.The relationship of phrase difference $(\varphi)$ and distance $(x)$ of two wavelengths is as follows:

$$
\varphi=\frac{4 \pi n_{0} x}{\lambda}
$$

Formula (1) shows the relationship between distance $(x)$ and phase difference $(\varphi)$ and $n_{0}\left(n_{0}=1\right)$ is the refractive index of air and $(\lambda)$ is the wavelength of light. The converter used in the experiment is a 12 -bit $\mathrm{A} / \mathrm{D}$ converter whose resolution is $1 / 2^{12}=1 / 4096 \approx 0.24 \%$. When the reference voltage is $5 \mathrm{~V}$, the minimum voltage change that can be distinguished is $5 \mathrm{~V} / 4096 \approx 1.2207 \mathrm{mV}$ so its corresponding voltage accuracy is $1.2207 \mathrm{mV}$ and the corresponding luminous flux (F) is $1.2207 \times 10^{-3} \mathrm{~lx}$. According to formula (2) which shows the conversion relation between luminous flux (F) and intensity of light (I), I is figured out as $I=3.0518 \times 10^{-4} \mathrm{~cd}$

$$
I=\frac{F}{4}
$$

Then according to formula (3),

$$
I \propto(1+\cos \varphi)
$$

Because the displacement calculated in the experiment is relative diaplacement, so assume

$$
I=(1+\cos \varphi)
$$

And then replace $\varphi$ into formula (1) so the accuracy of corresponding distance $(x)$ is $384.48 \mathrm{~nm}$.

The corresponding values of CCD of different wavelengths can be figured out by the use of reflective diffraction grafting and beam splitter[7]. Assume the voltage value generated by CCD on which the interference light with a wavelength of $\lambda$ strikes as $U$ and according to formula(4), what is figured out is

$$
\frac{U}{4}=(1+\cos \varphi)
$$

Replace formula(1) into formula(5), then

$$
x=\frac{\lambda \cdot \arccos \left(\frac{U}{4}-1\right)}{4 \pi}
$$

$x$ is the infinitesimal displacement of the sample and the value of $\lambda$ is got through the beam splitter. When the light strikes on the silicon chip of CCD, a potential well forms inside CCD because of the injection of photons so signal charges are generated. And the signal charges are output in the form of voltage values which is represented by U[8].

The Measuring Range.The wavelength of the selected light source SLD is $1550 \mathrm{~nm}$, power $10 \mathrm{~mW}$, bandwidth $40 \mathrm{~nm}$ and wavelength accuracy $100 \mathrm{GHz}$. Assume two threshold wavelengths $\lambda_{1}=1530 \mathrm{~nm}, \quad \lambda_{2}=1570 \mathrm{~nm}$, according to formula(1), formula(7) and formula(8) are got:

$$
\begin{aligned}
& \varphi_{1}^{-}=4 \pi \frac{x_{1}^{-}}{\lambda_{1}^{-}}=\varphi_{10}^{-}+2 a_{1}^{-} \pi \\
& \varphi_{1}^{+}=4 \pi \frac{x_{1}^{+}}{\lambda_{1}^{+}}=\varphi_{10}^{+}+2 a_{1}^{+} \pi
\end{aligned}
$$

$\varphi_{1}^{-}, \varphi_{1}^{+}$are the phase differences of the threshold wavelength accuracy and $\varphi_{10}^{-}, \varphi_{10}^{+}$are the decimal parts of $\varphi_{1}^{-}, \varphi_{1}^{+}$while $a_{1}^{-}, a_{1}^{+}$are the integer parts. As the two wavelengths are very close, when the sample is in the same place, $a_{1}^{-}$and $a_{1}^{+}$can be considered approximately equal, so the range $\mathrm{L}$ is got: 


$$
L_{1}=\frac{\lambda_{1}^{-} \lambda_{1}^{+}}{4 \pi\left(\lambda_{1}^{+}-\lambda_{1}^{-}\right)}\left(\varphi_{1}^{+}-\varphi_{1}^{-}\right)
$$

As $\varphi_{1}^{+}-\varphi_{1}^{-}$ranges from 0 to $2 \pi$, put the maximum value into formula (9),

$$
L_{1}=\frac{\lambda_{1}^{-} \lambda_{1}^{+}}{2\left(\lambda_{1}^{+}-\lambda_{1}^{-}\right)}
$$

In the same way,

$$
L_{2}=\frac{\lambda_{2}^{-} \lambda_{2}^{+}}{2\left(\lambda_{2}^{+}-\lambda_{2}^{-}\right)}
$$

As the range of $0.8 \mathrm{um} \sim 1.8 \mathrm{um}$ of the infrared band corresponds with the range of $375 \mathrm{THz} 167 \mathrm{THz}$ of frequency, according to formula (12),

$$
v=\lambda f
$$

we have $100 \mathrm{GHz}=0.2 \mathrm{~nm}$, so $\lambda_{1}=1530 \mathrm{~nm} \pm 0.2 \mathrm{~nm}, \quad \lambda_{2}=1570 \mathrm{~nm} \pm 0.2 \mathrm{~nm}$.

The CCD used in the system has 2048 pixels and the center wavelength of the system is $1550 \mathrm{~nm}$ so the minimum length change that can be distinguished by CCD is about $0.8 \mathrm{~nm}$. Put it into formula (11), we have $L_{2}=0.77 \mathrm{~mm}$, so the measuring range of the system is $0.77 \mathrm{~mm}$.

\section{Summary}

The system combines the reference arm with the feeler arm, reducing the interference caused by the optical path, optical fiber and coupler between the two arms under the separate condition. The probe of the system is designed on the basis of the wedge-shaped and endface-angled fiber-optical probe. Its output interference intensity changes with the change of the endface angle, achieving the adjustment of the reference signal and improving the effect of interference. The system, with high accuracy and wide range, at the same time, combining single optical fiber interference with the athermalization probe, although has imperfections, still has high research value.

\section{References}

[1]J. Yang, The Research of Two Double-beam Interferometric Optical Fiber Sensors [D], Shandong University,2008.

[2] Y. Q. Liu, The Research of The Online Measurement of Dual-wavelength Fiber Interference Step Height [D], Beijing Jiaotong University,2014.

[3] Dubrov, M.N.; Medvedev, P. V. Accurate laser interferometer system for displacement measurements with 1 pm resolution.Advanced Optoelectronics and Lasers, 2008.CAOL 2008.4th International Conference on.

[4]Y. F. Li, W. C. Wang, K.S. Lai, X. P. Liang, A Method to Realize The Coupling of Light Source and Optical Fiber [J], Physics and Engineering, 2003(01).

[5] Z. W. Wu, Y. Chen, X. H. Sun, The Research of The Optical Fiber Probe of The Coherent Imaging System of The Light With Common Path [M], Acta Photonica Sinica, 2011,(12):1899-1902.

[6] H. X. Wang, S. Y. Zhang, The principle and application of sensor (3rd edition) [M], Tianjin University Press, 20072:244-250.

[7]W. Z. Li, The Review of The Development and Application of The Optical Fiber Sensor [J], Application Technology, 2005,(7):153-154.

[8]Farooq, Muhammad. A comparison of image processing techniques for optical interference fringe analysis.Photonic Sensors, v 5, n 4, p 304-311, December 1, 2015. 